\title{
On the theory of discrete KMO-Langevin equations with reflection positivity (I)
}

To the memory of Professor Gishiro Maruyama

\author{
Yasunori OKABE*) \\ (Received October 15, 1986, Revised May 1, 1987)
}

\section{$\S 1$. Introduction}

In a series of papers $([6] \sim[10])$, the author has developed a theory of generalized Langevin equations for real continuous-time stationary Gaussian proccsses with reflection positivity. The time evolution of such a process $X$ $(t)$ can be described in terms of two kinds of Langevin equations with a notable difference in character of random forces $([9])$ : One is the first KMO-Langevin equation having a white noise as a random force, and it has a root in his study $([7])$ and $([8])$ of the $[\alpha, \beta, \gamma]$-Langevin equations. The other is the second KMO-Langevin equation where a colored noise named the Kubo noise is taken to be a random force.

With the linear response theory of R. Kubo ([4]) in mind, we established in [10] the fluctuation-dissipation theorems based on these Langevin equations of the two types; our discovery was that the classical Einstein relation for Ornstein-Uhlenbeck processes holds for the second type, but does not hold for the first one. In addition, we calculated the deviation from the classical Einstein relation. As a concrete example in physics, we discussed the Stokes-Boussinesque-Langevin equation with the Alder-Wainwright effect within our framework of the theory of KMO-Langevin equations possessing reflection positivity.

The purpose of the present and subsequent papers is to establish the discrete analogues of the results mentioned above for the first and second KMO-Langevin equations. Further development of these results will be discussed in the author's forthcomming third paper, with the same title. In contrast to the continuous-time case, we will find that the Einstein relation for discrete-time series $X(n)$ always deviates from the classical one in the Markovian case, not only for the first type (see $\S 7$ of the present (I )), but also for the second one (see $\S 6$ of (II)). In the third paper ([12]), we will discuss an entropy criterion and present an answer to the basic question in

*) Partially supported by the Grant-in-Aid for Scientific Research of the Ministry of Education, Science and Culture of Japan. 
the problem of modelling such as: "Which noise, white or Kubo, should be taken to be a random force in the equation with given coefficients $(\alpha, \beta, \gamma)$ called the KMO-Langevin data?". It should be noted that a variety of covariance functions arising from the theory of ARMA processes ([1]) and of one-dimensional transformations $([5])$ are all realized as those of real discrete-time stationary Gaussian processes with reflection positivity. And so they enter into the present framework of the theory of KMO-Langevin equations.

Now we will state the content of this paper. Let $\boldsymbol{X}=(X(n) ; n \in \boldsymbol{Z})$ be a real stationary Gaussian process with mean zero and covariance function $R$ :

$$
R(n)=E(X(n) X(0)) \quad(n \in \boldsymbol{Z}) .
$$

In $\S 2$ we will briefly recall the spectral theory of $\boldsymbol{X}$. In particular, for the Hardy spectral density $\Delta$ of $\boldsymbol{X}$ such that $\log \Delta \in L^{1}((-\pi, \pi))$, we define the outer function $h$ of $\boldsymbol{X}$ on $U_{1}(0) \equiv\{z \in \boldsymbol{C} ;|z|<1\}$ by

$$
h(z)=\exp \left(\frac{1}{4 \pi} \int_{-\pi}^{\pi} \frac{e^{i \theta}+z}{e^{i \theta}-z} \log \Delta(\theta) d \theta\right) .
$$

In a similar manner to the continuous-time case discussed in [6], we will in $\S 3$ define the reflection positivity of $\boldsymbol{X}$, which can be characterized by the condition: there exists a bounded Borel measure $\sigma$ on $[-1,1]$ such that

$$
R(n)=\int_{[-1,1]} t^{|n|} \sigma(d t) \quad(n \in \boldsymbol{Z}) .
$$

The following conditions are assumed in what follows:

$$
\begin{aligned}
& \sigma(\{-1,1\})=0 \\
& \int_{-1}^{1}\left(\frac{1}{1+t}+\frac{1}{1-t}\right) \sigma(\mathrm{d} t)<\infty .
\end{aligned}
$$

From these conditions it follows that

$$
R \in l^{1}(\boldsymbol{Z}) .
$$

Section 4 is devoted to the study of the structure of the outer function $h$ as well as of the canonical representation kernel $E=\hat{h}$, which will play an important role in this paper. By using the result for the continuous-time case obtained in [9], we have

THEOREM 4.1. There exists a unique triple $\left(\alpha_{1}, \beta_{1}, \rho_{1}\right)$ such that

(i) $\alpha_{1}>0$ and $\beta_{1}>0$

(ii) $\rho_{1}$ is a bounded Borel measure on $[-1,1]$ with $\rho_{1}(\{-1,1\})=0$ 
(iii) for any $z \in U_{1}(0)$

$$
h(z)=\frac{\alpha_{1}}{\sqrt{2 \pi}} \frac{1}{\beta_{1}(1+z)+1-z+\left(1-z^{2}\right) \int_{-1}^{1} \frac{1}{1-t z} \rho_{1}(d t)} .
$$

We call the triple $\left(\alpha_{1}, \beta_{1}, \rho_{1}\right)$ in Theorem 4.1 the first KMO-Langevin data associated with $\sigma$ (or $R$ ). Another triple $\left(\alpha_{1}, \beta_{1}, \gamma_{1}\right)$ is also called by the same name, where $\gamma_{1}$ is a function on $\boldsymbol{Z}$ defined by

$$
\gamma_{1}=\frac{1}{2 \pi}\left(\left(1-e^{2 i \cdot}\right) \int_{-1}^{1} \frac{1}{1-t e^{i \cdot} \cdot \rho_{1}}(d t)\right)^{\wedge} .
$$

More explicitly,

$$
\gamma_{1}(n)= \begin{cases}0 & \text { for } n \in\{-1,-2, \ldots\} \\ \int_{-1}^{1} t^{n} \rho_{1}(d t) & \text { for } n \in\{0,1\} \\ \int_{-1}^{1} t^{n-2}\left(t^{2}-1\right) \rho_{1}(d t) & \text { for } n \in\{2,3, \ldots\},\end{cases}
$$

which implies that

$$
\gamma_{1} \in l^{1}(\boldsymbol{Z}) .
$$

It will be found in $\S 5$ that the correspondence between $\sigma$ and $\left(\alpha_{1}, \beta_{1}, \rho_{1}\right)$ is bijective (Theorem 5.1). Furthermore we will obtain an explicit formula of the triple $\left(\alpha_{1}, \beta_{1}, \gamma_{1}\right)$ in terms of $\sigma$ (Theorem 5.2).

By using Theorem 4.1, we will in $\S 6$ derive a stochastic difference equation with a white noise as its random force describing the time evolution of $\boldsymbol{X}$.

THEOREM 6.1.

$$
\begin{array}{r}
X(n)-X(n-1)=-\beta_{1}(X(n)+X(n-1))-\left(\gamma_{1} * X\right)(n)+\alpha_{1} \xi(n) \\
\text { a. s. }(n \in \boldsymbol{Z}),
\end{array}
$$

where $\boldsymbol{\xi}=(\boldsymbol{\xi}(n) ; n \in \boldsymbol{Z})$ is a real Gaussian white noise.

This equation (1.10) is nothing but the first KMO-Langevin equation we are looking for in the discrete-time case. Conversely, we will show that the first KMO-Langevin equation can be uniquely solved for any given triple $\left(\alpha_{1}, \beta_{1}, \rho_{1}\right)$ with conditions (i ) and (ii) in Theorem 4.1 and a real Gaussian white noise $\boldsymbol{\xi}$ (Theorem 6.2).

Fundamental examples of $\boldsymbol{X}$ are given by the Markov processes $\boldsymbol{X}_{p}$, $-1<p<1$, with covariance functions $R_{p}$ :

$$
R_{p}(n)=p^{|n|} \quad(n \in \boldsymbol{Z}) .
$$


It then follows that the outer function $h_{p}$ of $\boldsymbol{X}_{p}$ becomes

$$
h_{p}(z)=\frac{1}{\sqrt{2 \pi}} \frac{\sqrt{1-p^{2}}}{1-p z} \quad\left(z \in U_{1}(0)\right) .
$$

By rewriting it in the form (iii) in Theorem 4.1, we have

$$
h_{p}(z)=\frac{\alpha_{p}^{(1)}}{\sqrt{2 \pi}} \frac{1}{\beta_{p}^{(1)}(1+z)+1-z},
$$

where

$$
\alpha_{p}^{(1)}=2 \sqrt{\frac{1-p}{1+p}} \text { and } \beta_{p}^{(1)}=\frac{1-p}{1+p} .
$$

Therefore, we find from Theorem 6.1 that the time evolution of $\boldsymbol{X}_{p}$ is governed by

$$
\begin{array}{r}
X_{p}(n)-X_{p}(n-1)=-\beta_{p}^{(1)}\left(X_{p}(n)+X_{p}(n-1)\right)+\alpha_{p}^{(1)} X_{0}(n) \\
\text { a.s. }(n \in \boldsymbol{Z}) .
\end{array}
$$

We note that $\boldsymbol{X}_{0}$ is a white noise.

Concerning a discrete analogue of the generalized fluctuation-dissipation theorem for the continuous-time case discussed in [10], we will in $\S 7$ show

THEOREM 7.1.

(i) For any $\theta \in(-\pi, \pi)$

$$
\frac{1}{\beta_{1}\left(1+e^{i \theta}\right)+1-e^{i \theta}+2 \pi \tilde{\gamma}_{1}(\theta)}=\frac{h\left(e^{i \theta}\right)}{2 \lim _{\tau \downarrow-\pi} h\left(e^{i \tau}\right)}
$$

(ii) $\frac{\alpha_{1}^{2}}{2}=R(0) C_{\beta_{1}, \gamma_{1}}$,

where

$$
C_{\beta_{1}, \gamma_{1}}=\pi\left(\int_{-\pi}^{\pi}\left|\beta_{1}\left(1+e^{i \theta}\right)+1-e^{i \theta}+2 \pi \tilde{\gamma}_{1}(\theta)\right|^{-2} d \theta\right)^{-1}
$$

(iii) $D=\frac{\alpha_{1}^{2}}{2\left(2 \beta_{1}\right)^{2}}$,

where

$$
D=\lim _{N \rightarrow \infty} \frac{1}{2 N} E\left(\left(\sum_{n=0}^{N} X(n)\right)^{2}\right) .
$$

(iv) $D=\frac{R(0)}{2 \beta_{1}} \frac{C_{\beta_{1}, \gamma_{1}}}{2 \beta_{1}}$

(v) $\frac{C_{\beta_{1}, \gamma_{1}}}{2 \beta_{1}}-1=\frac{1}{R(0)} \int_{-1}^{1} \int_{-1}^{1} \frac{1+t}{1-t u} \sigma(d t) \rho_{1}(d u)$. 
By taking account of the physical meaning of the generalized fluctuation-dissipation theorem for the continuous-time case given in [10], we call the relations ( $i$ ), (ii) and (iv) in Theorem 7.1 the generalized first fluctuation-dissipation theorem, the generalized second fluctuationdissipation theorem and the generalized Einstein relation, respectively. And we call the constants $C_{\beta_{1}, \gamma_{1}}$ in (1.15) and $D$ in (1.16) the generalized friction coefficient and the diffusion constant of $\boldsymbol{X}$, respectively. We note that this $D$ is also expressed in terms of $R$ :

$$
D=\sum_{n=0}^{\infty} R(n)-\frac{R(0)}{2} .
$$

In particular, the diffusion constant $D_{p}$ of $\boldsymbol{X}_{p}$ is given by

$$
D_{p}=\frac{R_{p}(0)}{2 \beta_{p}^{(1)}}\left(=\frac{1+p}{2(1-p)}\right) \quad(p \in(-1,1)) .
$$

This expression is the classical Einstein relation. And so we conclude from the generalized Einstein relation (iv) in Theorem 7.1 that there occures a deviation from the classical Einstein relation (1.18) in general nonMarkovian cases. Such a deviation can be calculated explicitly by the formula ( $\mathrm{v}$ ) in Theorem 7.1.

The author would like to thank the referees for their valuable and constructive advices.

\section{$\S 2$. Preliminaries}

Let $\boldsymbol{X}=(X(n) ; n \in \boldsymbol{Z})$ be a real stationary Gaussian process with discrete time on a probability space $(\Omega, \mathscr{F}, P)$ and let $R$ be its covariance function :

$$
R(n)=E(X(n) X(0)) \quad(n \in \boldsymbol{Z}) .
$$

We assume that the spectral measure of $\boldsymbol{X}$ has a spectral density $\Delta=\Delta(\theta)$ such that

(2.2) $\log \Delta \in L^{1}((-\pi, \pi))$,

(2.3) $\quad R(n)=\int_{-\pi}^{\pi} e^{-i n \theta} \Delta(\theta) d \theta$.

Then we can define an outer function $h$ of $\Delta$ by

$$
h(z)=\exp \left\{\frac{1}{4 \pi} \int_{-\pi}^{\pi} \frac{e^{i \theta}+z}{e^{i \theta}-z} \log \Delta(\theta) d \theta\right\}
$$

for $z \in U_{1}(0)=\{z \in C ;|z|<1\}$. Such a function $h$ has the following prop- 
erties $([2])$ :

$$
\begin{array}{ll}
\sup _{0<r<1} \int_{-\pi}^{\pi}\left|h\left(r e^{i \theta}\right)\right|^{2} d \theta<\infty ; & \\
h\left(e^{i \theta}\right)=\lim _{r \uparrow 1} h\left(r e^{i \theta}\right) \text { exists } & \text { a.e. } \theta \in(-\pi, \pi) ; \\
\left|h\left(e^{i \theta}\right)\right|^{2}=\Delta(\theta) & \text { a.e. } \theta \in(-\pi, \pi) ; \\
\lim _{r+1} h\left(r e^{i \cdot}\right)=h\left(e^{i \cdot}\right) & \text { in } L^{2}((-\pi, \pi)) .
\end{array}
$$

Next we define a function $E$ on $\boldsymbol{Z}$ by

$$
E(n)=\hat{h}(n)=\int_{-\pi}^{\pi} e^{-i n \theta} h\left(e^{i \theta}\right) d \theta .
$$

It then follows from the above properties of $h$ that

(2.10) $E \in l^{2}(\boldsymbol{Z})$,

(2.11) $E(n)=0 \quad(n=-1,-2,-3, \ldots)$,

(2.12) $\lim _{n \rightarrow \infty} E(n)=0$, and

$$
R(n)=\frac{1}{2 \pi} \sum_{m=0}^{\infty} E(|n|+m) E(m) \quad(n \in \boldsymbol{Z}) .
$$

As is well known $([3])$, there exists a normalized Gaussian white noise $\boldsymbol{\xi}=(\boldsymbol{\xi}(n) ; n \in \boldsymbol{Z})$ such that

$$
\begin{aligned}
& X(n)=\frac{1}{\sqrt{2 \pi}} \sum_{m=-\infty}^{n} E(n-m) \xi(m) \quad \text { in } L^{2}(\Omega, \mathscr{F}, P) . \\
& \sigma(X(m) ; m \leq n)=\sigma(\boldsymbol{\xi}(m) ; m \leq n) \quad(n \in \boldsymbol{Z}) .
\end{aligned}
$$

By taking account of what have been obtained so far, the function $E$ is said to be a canonical representation kernel of $\boldsymbol{X}$.

\section{$\S 3$. Reflection positivity}

Let $\boldsymbol{X}=(X(n) ; n \in \boldsymbol{Z})$ be a real stationary Gaussian process on $(\Omega, \mathscr{F}$, $P$ ) with covariance function $R$. We denote by $\boldsymbol{M}$ (resp. $\boldsymbol{M}^{+}$) the closed linear hull of $\{X(n) ; n \in Z$ ) (resp. $\{X(n) ; n \geq 0\})$ in $L^{2}(\Omega, \mathscr{F}, P)$ and by $P_{\boldsymbol{M}^{+}}$the orthogonal projection on $\boldsymbol{M}^{+}$. The time reflection operator $T$ is a unitary and self-adjoint operator on $\boldsymbol{M}$ defined by

$$
T(X(n))=X(-n) \quad(n \in \boldsymbol{Z}) .
$$

As in the continuous-time case $([6])$, we say that $\boldsymbol{X}$ has reflection positivity (T-positivity) if and only if $P_{M^{+}} T P_{M^{+}}$is non-negative.

In the sequel, we assume that $\boldsymbol{X}$ has reflection positivity. By taking the same consideration as $\S 2$ in [6], we see that there exists a unique Borel 
measure $\sigma$ on $[-1,1]$ such that

$$
R(n)=\int_{[-1,1]} t^{|n|} \sigma(d t) \quad(n \in \boldsymbol{Z}) .
$$

EXAmPle 3.1 For each $p \in(-1,1)$ consider a non-negative definite function $R_{p}$ corresponding to the case $\sigma=\delta_{\{p\}}$, i. e.,

$$
R_{p}(n)=p^{|n|} \text {. }
$$

Then the spectral density $\Delta_{p}$ of $R_{p}$ is given by

$$
\Delta_{p}(\theta)=\frac{1}{2 \pi} \frac{1-p^{2}}{\left|1-p e^{i \theta}\right|^{2}} \quad(\theta \in(-\pi, \pi)) .
$$

We now impose the additional assumption on the measure $\sigma$ in (3.1):

(3.4) $\sigma(\{-1,1\})=0$.

Then it immediately follows that the spectral measure of $R$ has the density $\Delta$ of the form

$$
\Delta(\theta)=\frac{1}{2 \pi} \int_{-1}^{1} \frac{1-t^{2}}{\left|1-t e^{i \theta}\right|^{2}} \sigma(d t) \quad(\theta \in(-\pi, \pi)) .
$$

By using a homeomorphism $\varphi$ from $(-1,1]$ onto $[0, \infty)$ :

$$
\text { (3.6) } \varphi(t)=\frac{1-t}{1+t} \text {, }
$$

we define a bounded Borel measure $\sigma_{c}$ on $[0, \infty)$ by

(3.7) $\sigma_{c}=\varphi(\sigma)$.

A direct calculation yields that

$$
\Delta\left(2 \tan ^{-1} \xi\right)=\frac{1+\xi^{2}}{2 \pi} \int_{0}^{\infty} \frac{\lambda}{\xi^{2}+\lambda^{2}} \sigma_{c}(d \lambda) \quad(\xi \in \boldsymbol{R}),
$$

which leads us to consider

$$
\Delta_{c}(\xi)=\frac{1}{\pi} \int_{0}^{\infty} \frac{\lambda}{\xi^{2}+\lambda^{2}} \sigma_{c}(d \lambda) .
$$

Since $\sigma_{c}(\{0\})=0$, it follows from Lemma 2.12 in [6] that $\Delta_{c}$ is a Hardy weight, that is,

$$
\frac{\log \Delta_{c}(\boldsymbol{\xi})}{1+\xi^{2}} \in L^{1}(\boldsymbol{R})
$$

And so we can define an outer function $h_{c}$ of $\Delta_{c}$ on $C^{+}$by 


$$
h_{c}(\xi)=\exp \left\{\frac{1}{2 \pi i} \int_{R} \frac{1+\xi \xi}{\xi-\xi} \frac{\log \Delta_{c}(\xi)}{1+\xi^{2}} d \xi\right\}
$$

Noting that

$$
\int_{-\pi}^{\pi}|\log \Delta(\theta)| d \theta=2 \int_{\boldsymbol{R}}\left|\frac{\log \Delta\left(2 \tan ^{-1} \xi\right)}{1+\xi^{2}}\right| d \xi,
$$

we see from (3.8), (3.9) and (3.10) that the spectral density $\Delta$ of $R$ satisfies condition (2.2). By a direct calculation, the outer function $h$ of $\Delta$ can be rewritten into the form

$$
h(z)=\exp \left\{\frac{1}{2 \pi i} \int_{R} \frac{1+\lambda \xi}{\lambda-\xi} \frac{\log \Delta\left(2 \tan ^{-1} \lambda\right)}{1+\lambda^{2}} d \lambda\right\},
$$

where $z \in U_{1}(0)$ and $\xi=i \frac{1-z}{1+z} \in C^{+}$.

Since for any $\xi \in C^{+}$

$$
\exp \left\{\frac{1}{2 \pi i} \int_{R} \frac{1+\xi \xi}{\xi-\xi} \frac{\log \left(2 / 1+\xi^{2}\right)}{1+\xi^{2}} d \xi\right\}=\frac{\sqrt{2}}{1-i \xi},
$$

it follows from (3.8), (3.9), (3.11) and (3.12) that

IEMMA 3.1.

(i) $h(z)=\frac{\sqrt{2}}{1+z} h_{c}\left(i \frac{1-z}{1+z}\right) \quad\left(z \in U_{1}(0)\right)$

(ii) $h_{c}(\xi)=\frac{\sqrt{2}}{1-i \xi} h\left(\frac{i-\xi}{i+\xi}\right) \quad\left(\xi \in C^{+}\right)$.

Furthermore, immediately from (3.7), we obtain

LEMMA 3.2.

$$
\begin{aligned}
& \text { (i) } \int_{-1}^{1} \frac{1+t}{1-t} \sigma(d t)=\int_{0}^{\infty} \lambda^{-1} \sigma_{c}(d \lambda) \\
& \text { (ii) } \int_{-1}^{1} \frac{1-t}{1+t} \sigma(d t)=\int_{0}^{\infty} \lambda \sigma_{c}(d \lambda) .
\end{aligned}
$$

\section{$\S 4$. Outher function $h$ and canonical representation kernel $E$}

Let $\boldsymbol{X}=(X(n) ; n \in \boldsymbol{Z})$ be a real stationary Gaussian process on $(\Omega, \mathscr{F}$, $P$ ) satisfying reflection positivity. It then follows that the covariance function $R$ of $\boldsymbol{X}$ has such a representation as (3.1) with a bounded Borel measure $\sigma$ on $[-1,1]$. In the sequel, we assume the following conditions :

$$
\begin{aligned}
& \sigma(\{-1,1\})=0 \\
& \int_{-1}^{1}\left(\frac{1}{1+t}+\frac{1}{1-t}\right) \sigma(\mathrm{d} t)<\infty .
\end{aligned}
$$


At first we will show the following structure theorem for the outer function $h$ of $\boldsymbol{X}$, which will be the key to derive a description of the time evolution of $\boldsymbol{X}$ in $\S 6$.

THEOREM 4.1. There exists a unique triple $\left(\alpha_{1}, \beta_{1}, \rho_{1}\right)$ such that

(i) $\alpha_{1}>0$ and $\beta_{1}>0$

(ii) $\rho_{1}$ is a bounded Borel measure on $[-1,1]$ with $\rho_{1}(\{-1,1\})=0$

(iii) for any $z \in U_{1}(0)$

$$
h(z)=\frac{\alpha_{1}}{\sqrt{2 \pi}} \frac{1}{\beta_{1}(1+z)+1-z+\left(1-z^{2}\right) \int_{-1}^{1} \frac{1}{1-t z} \rho_{1}(d t)} .
$$

Proof. Since it follows from Lemma 3.2 and condition (4.2) that $\int_{0}^{\infty}\left(\lambda^{-1}+\lambda\right) \sigma_{c}(d \lambda)<\infty$, we can apply Theorem 2.2 in [9] to see that there exists a unique triple $\left(\alpha_{c}, \beta_{c}, \rho_{c}\right)$ such that

$$
\alpha_{c}>0 \text { and } \beta_{c}>0
$$

(4.4) $\quad \rho_{c}$ is a Borel measure on $[0, \infty)$ satisfying

$$
\rho_{c}(\{0\})=0 \text { and } \int_{0}^{\infty} \frac{1}{1+\lambda} \rho_{c}(d \lambda)<\infty
$$

$$
h_{c}(\xi)=\frac{\alpha_{c}}{\sqrt{2 \pi}} \frac{1}{\beta_{c}-i \xi-i \xi \int_{0}^{\infty} \frac{1}{\lambda-i \xi} \rho_{c}(d \lambda)} \quad \text { for any } \xi \in C^{+} \text {. }
$$

Then we define a triple $\left(\alpha_{1}, \beta_{1}, \rho_{1}\right)$ by

(4.6) $\quad \alpha_{1}=\sqrt{2} \alpha_{c}$

(4.7) $\quad \beta_{1}=\beta_{c}$

$$
\rho_{1}(d t)=\frac{1+t}{2}\left(\varphi^{-1} \rho_{c}\right)(d t) .
$$

In particular, we have

$$
\rho_{1}([-1,1])=\int_{0}^{\infty} \frac{1}{\lambda+1} \rho_{c}(d \lambda) .
$$

Therefore, we find that the triple $\left(\alpha_{1}, \beta_{1}, \rho_{1}\right)$ satisfies (i) and (ii) in Theorem 4.1. Furthermore, it follows from Lemma 3.1 (i ) and (4.5) that for any $z \in U_{1}(0)$

$$
h(z)=\frac{\sqrt{2}}{1+z} \frac{\alpha_{c}}{\sqrt{2 \pi}} \frac{1}{\beta_{c}+\frac{1-z}{1+z}+\frac{1-z}{1+z} \int_{0}^{\infty}\left(\lambda+\frac{1-z}{1+z}\right)^{-1} \rho_{c}(d \lambda)} .
$$


On the other hand, by the definition of $\varphi$,

$$
\begin{aligned}
\int_{0}^{\infty}\left(\lambda+\frac{1-z}{1+z}\right)^{-1} \rho_{c}(d \lambda) & =\int_{-1}^{1}\left(\frac{1-t}{1+t}+\frac{1-z}{1+z}\right)^{-1}\left(\varphi^{-1} \rho_{c}\right)(d t) \\
& =(1+z) \int_{-1}^{1} \frac{1}{1-t z} \frac{1+t}{2}\left(\varphi^{-1} \rho_{c}\right)(d t)
\end{aligned}
$$

Therefore, we find from (4.6), (4.7) and (4.8) that the triple $\left(\alpha_{1}, \beta_{1}, \rho_{1}\right)$ satisfies relation (iii) in Theorem 4.1.

The uniqueness of such a triple $\left(\alpha_{1}, \beta_{1}, \rho_{1}\right)$ can be proved as follows: since $0 \leq \frac{(1+x)(1-x)}{1-t x} \leq 2$ for any $t, x \in(-1,1)$, we see that

(4.10) $\lim _{x \downarrow-1} h(x)=\frac{\alpha_{1}}{2 \sqrt{2 \pi}}$

and

(4.11) $\lim _{x+1} h(x)=\frac{\alpha_{1}}{2 \sqrt{2 \pi} \beta_{1}}$,

which determine uniquely the pair $\left(\alpha_{1}, \beta_{1}\right)$. Furthermore, since

$$
\int_{-1}^{1} \frac{1}{1+x t} \rho_{1}(d t)=\frac{1}{x} \int_{0}^{1} \frac{1}{\frac{1-x}{x}+s} \rho_{1}(d(s-1)) \text { for any } x \in(0,1),
$$

it follows from the uniqueness of Stieltjes transform that the measure $\rho_{1}$ is uniquely determined.

(Q. E. D.)

Next we will show the following expression of $h$, closely related to the one established in Theorem 4.1.

THEOREM 4.2. There exists a unique Borel measure $\nu$ on $[-1,1]$ such that

(i ) $\boldsymbol{\nu}(\{-1,1\})=0$ and $\boldsymbol{\nu}([-1,1])<\infty$

(ii) $\int_{-1}^{1}\left(\frac{1}{1-t}+\frac{1}{1+t}\right) \nu(d t)<\infty$

(iii) $h(z)=\frac{1}{2 \pi} \int_{-1}^{1} \frac{1}{1-t z} \nu(d t) \quad$ for any $z \in U_{1}(0)$.

Proof. By Theorem 2.1 and (2.19) in [9], we see that there exists a unique Borel measure $\nu_{c}$ on $[0, \infty)$ such that

$$
\begin{aligned}
& \nu_{c}(\{0\})=0 \text { and } \nu_{c}([0, \infty])<\infty \\
& \int_{0}^{\infty} \lambda^{-1} \nu_{c}(d \lambda)<\infty
\end{aligned}
$$




$$
h_{c}(\xi)=\frac{1}{2 \pi} \int_{0}^{\infty} \frac{1}{\lambda-i \xi} \nu_{c}(d \lambda) \quad \text { for any } \xi \in C^{+} .
$$

Then we define a Borel measure $\nu$ on $[-1,1]$ by

$$
\nu(d t)=\frac{1+t}{\sqrt{2}}\left(\varphi^{-1} \nu_{c}\right)(d t) .
$$

Since

$$
\begin{aligned}
\int_{0}^{\infty} \lambda^{-1} \nu_{c}(d \lambda) & =\int_{-1}^{1}(\varphi(t))^{-1}\left(\varphi^{-1} \nu_{c}\right)(d t) \\
& =\sqrt{2} \int_{-1}^{1} \frac{1}{1-t} \nu(d t),
\end{aligned}
$$

it follows from (4.12), (4.13) and (4.15) that the measure $\nu$ satisfies (i ) and (ii) in Theorem 4.2. Furthermore, we see from Lemma 3.1 (i) and (4.14) that for any $z \in U_{1}(0)$

$$
\begin{aligned}
h(z) & =\frac{\sqrt{2}}{1+z} \frac{1}{2 \pi} \int_{0}^{\infty}\left(\lambda+\frac{1-z}{1+z}\right)^{-1} \nu_{c}(d \lambda) \\
& =\frac{\sqrt{2}}{1+z} \frac{1}{2 \pi} \int_{-1}^{1}\left(\frac{1-t}{1+t}+\frac{1-z}{1+z}\right)^{-1}\left(\varphi^{-1} \nu_{c}\right)(d t) \\
& =\frac{1}{2 \pi} \int_{-1}^{1} \frac{1}{1-t z} \frac{1+t}{\sqrt{2}}\left(\varphi^{-1} \nu_{c}\right)(d t),
\end{aligned}
$$

which, together with (4.15), yields (iii) in Theorem 4.2. The uniqueness of a Borel measure $\boldsymbol{\nu}$ satisfying ( i ), (ii) and (iii) can be proved by using the uniqueness of Stieltjes transform.

By taking a boundary value of the outer function $h$, we can rephrase Theorems 4.1 and 4.2 as follows.

Corollary 4.1. For almost all $\theta \in(-\pi, \pi)$,

(i) $h\left(e^{i \theta}\right)=\frac{\alpha_{1}}{\sqrt{2 \pi}} \frac{1}{\beta_{1}\left(1+e^{i \theta}\right)+1-e^{i \theta}+\left(1-e^{2 i \theta}\right) \int_{-1}^{1} \frac{1}{1-t e^{i \theta}} \rho_{1}(d t)}$

(ii) $h\left(e^{i \theta}\right)=\frac{1}{2 \pi} \int_{-1}^{1} \frac{1}{1-t e^{i \theta}} \nu(d t)$.

As a consequence of the above expression (ii) of $h$, we can obtain an expression of the canonical representation kernel $E$ in (2.9), which says that $E(n), n \geq 0$, is nothing but the moment sequence of the measure $\nu$.

THEOREM 4. 3 .

$$
E(n)=\chi_{[0, \infty)}(n) \int_{-1}^{1} t^{n} \nu(d t) \quad \text { for any } n \in \boldsymbol{Z} .
$$


Proof. By Theorem 4.2 (ii) and Corollary 4.1 (ii), the following series

$$
h\left(e^{i \theta}\right)=\frac{1}{2 \pi} \sum_{n=0}^{\infty} e^{i \theta n}\left(\cdot \int_{-1}^{1} t^{n} \nu(d t)\right)
$$

is absolutely convergent (a.e.). Therefore, by (2.9), we have the assertion.

Concerning a relation between the measures $\sigma$ in (3.1) and $\nu$ in Theorem 4.2 , we will show

THEOREM 4. 4.

$$
\sigma(d t)=\frac{1}{2 \pi}\left(\int_{-1}^{1} \frac{1}{1-t s} \nu(d s)\right) \nu(d t) .
$$

Proof. By Theorem 4.3, for any $n \in\{0,1,2, \ldots\}$,

$$
\sum_{m=0}^{\infty} E(n+m) E(m)=\int_{-1}^{1} t^{n}\left(\int_{-1}^{1} \frac{1}{1-t s} \nu(d s)\right) \nu(d t) .
$$

Therefore, by (2.14) and (3.1), we have Theorem 4.4.

(Q.E. D.)

Remark 4.1. By Lemma 2.6 (i) in [9], the measure $\sigma_{c}$ in $(3.7)$ is related to the measure $\nu_{c}$ in (4.12) as follows:

$$
\sigma_{c}(d \lambda)=\frac{1}{2 \pi}\left(\int_{0}^{\infty} \frac{1}{\lambda+\lambda^{\prime}} \nu_{c}\left(d \lambda^{\prime}\right)\right) \nu_{c}(d \lambda)
$$

In view of (3.7) and (4.15), it turns out that this equality is equivalent to (4. 16).

Example 4.1. For each fixed $p \in(-1,1)$, consider the non-neagtive definite function $R_{p}$ in (3.2). Then we see that the outer function $h_{p}$ and the canonical representation kernel $E_{p}$ become

$$
\begin{array}{ll}
h_{p}(z)=\frac{1}{\sqrt{2 \pi}} \frac{\sqrt{1-p^{2}}}{1-p z} & \left(z \in U_{1}(0)\right) \\
E_{p}(n)=\chi_{[0, \infty)}(n) \sqrt{2 \pi\left(1-p^{2}\right)} p^{n} & (n \in \boldsymbol{Z}) .
\end{array}
$$

Furthermore we observe that

$$
h_{p}(z)=\frac{1}{\sqrt{2 \pi}} 2 \sqrt{\frac{1-p}{1+p}} \frac{1}{\frac{1-p}{1+p}(1+z)+1-z} \quad\left(z \in U_{1}(0),\right.
$$

which implies that the triple $\left(\alpha_{p}^{(1)}, \beta_{p}^{(1)}, \rho_{p}^{(1)}\right)$ in Theorem 4.1 associated with $R_{p}$ becomes 
(4.21) $\quad \alpha_{p}^{(1)}=2 \sqrt{\frac{1-p}{1+p}}, \beta_{p}^{(1)}=\frac{1-p}{1+p}$ and $\rho_{p}^{(1)}=0$.

EXAMPLE 4.2. Let $R$ be the non-negative definite function given by (4.22) $\quad R(n)=\sigma_{1} p_{1}^{|n|}+\sigma_{2} p_{2}^{|n|} \quad(n \in \boldsymbol{Z})$, where $\sigma_{1}, \sigma_{2}>0$ and $-1<p_{1}<p_{2}<1$. Put

$$
\begin{array}{ll}
(4.23) & a_{1}=\sigma_{1}\left(1-p_{1}^{2}\right) p_{2}+\sigma_{2}\left(1-p_{2}^{2}\right) p_{1} \\
(4.24) & a_{2}=\sigma_{1}\left(1-p_{1}^{2}\right)\left(1+p_{2}^{2}\right)+\sigma_{2}\left(1-p_{2}^{2}\right)\left(1+p_{1}^{2}\right) .
\end{array}
$$

By a direct calculation, we can conclude that the outer function $h$, the triple $\left(\alpha_{1}, \beta_{1}, \rho_{1}\right)$ and the measure $\nu$ associated with this $R$ are given as follows:

(i) The case $a_{1}=0\left(-1<p_{1}<0<p_{2}<1\right)$ :

$$
\begin{aligned}
& h(z)=\sqrt{\frac{a_{2}}{2 \pi}} \frac{1}{\left(1-p_{1} z\right)\left(1-p_{2} z\right)} \quad\left(z \in\left(U_{1}(0)\right)\right. \\
& \left\{\begin{array}{l}
\alpha_{1}=\frac{2 \sqrt{a_{2}}}{\left(1+p_{1}\right)\left(1+p_{2}\right)} \\
\beta_{1}=\frac{\left(1-p_{1}\right)\left(1-p_{2}\right)}{\left(1+p_{1}\right)\left(1+p_{2}\right)} \\
\rho_{1}(d t)=\frac{-2 p_{1} p_{2}}{\left(1+p_{1}\right)\left(1+p_{2}\right)} \delta_{\{0\}}(d t) \\
\nu=\frac{\sqrt{2 \pi a_{2}}}{p_{2}-p_{1}}\left\{\left(-p_{1}\right) \delta_{\left\{p_{1}\right\}}+p_{2} \delta_{\left\{p_{2}\right\}}\right\}
\end{array}\right.
\end{aligned}
$$

(ii) The case $a_{1} \neq 0$ :

$$
h(z)=\sqrt{\frac{r_{1}}{2 \pi}} \frac{1-q_{1} z}{\left(1-p_{1} z\right)\left(1-p_{2} z\right)} \quad\left(z \in U_{1}(0)\right)
$$

$$
\begin{aligned}
& \text { (4.29) } \quad\left\{\begin{array}{l}
\alpha_{1}=\frac{2 \sqrt{r_{1}}\left(1+q_{1}\right)}{\left(1+p_{1}\right)\left(1+p_{2}\right)} \\
\beta_{1}=\frac{\left(1+q_{1}\right)\left(1-p_{1}\right)\left(1-p_{2}\right)}{\left(1-q_{1}\right)\left(1+p_{1}\right)\left(1+p_{2}\right)} \\
\rho_{1}(d t)=\frac{2\left(q_{1}-p_{1}\right)\left(p_{2}-q_{1}\right)}{\left(1-q_{1}\right)\left(1+p_{1}\right)\left(1+p_{2}\right)} \delta_{\left\{q_{1}\right\}}(d t)
\end{array}\right. \\
& (4.30) \quad \nu=\frac{\sqrt{2 \pi r_{1}}}{p_{2}-p_{1}}\left\{\left(q_{1}-p_{1}\right) \delta_{\left\{p_{1}\right\}}+\left(p_{2}-q_{1}\right) \delta_{\left\{p_{2}\right\}}\right\},
\end{aligned}
$$

where

$$
q_{1}=\frac{1}{2}\left(\frac{a_{2}}{a_{1(+)}} \sqrt{\left(\frac{a_{2}}{a_{1}}\right)^{2}-4}\right) \text { if } a_{1}>0
$$$$
\text { (4.32) } \quad r_{1}=\frac{a_{1}}{q_{1}} \text {. }
$$ 
We note that $p_{1}<q_{1}<p_{2}$ and $q_{1}>0$ if $a_{1}>0$.

\section{§5. The first KMO-Langevin data}

This section is devoted to the further study of the key expression of $h$ in Theorem 4. 1. In order to investigate the correspondence between $\sigma$ in (3.1) and $\left(\alpha_{1}, \beta_{1}, \rho_{1}\right)$ in Theorem 4.1, we introduce two classes $\Sigma_{1}$ and $\mathscr{L}_{1}$ by

(5.1) $\Sigma_{1}=\{\sigma ; \sigma$ is a bounded Borel measure on $[-1,1]$ such that

$$
\left.\sigma(\{-1,1\})=0 \text { and } \int_{-1}^{1}\left(\frac{1}{1+t}+\frac{1}{1-t}\right) \sigma(d t)<\infty\right\}
$$

and

(5.2) $\mathscr{L}_{1}=\{(\alpha, \beta, \rho) ; \alpha>0, \beta>0$ and $\rho$ is a bounded Borel measure on $[-1,1]$ such that $\rho(\{-1,1\})=0\}$.

For each $\sigma \in \Sigma_{1}$, we denote by $R_{\sigma}, \Delta_{\sigma}$ and $h_{\sigma}$ the non-negative definite function, the spectral density and the outer function associated with $\sigma$, respectively:

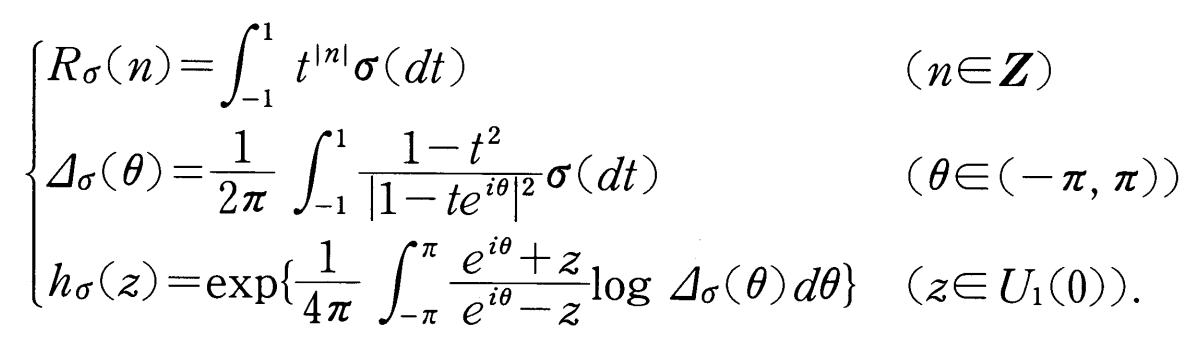

THEOREM 5.1. There exists a bijective mapping $L_{1}$ from $\Sigma_{1}$ onto $\mathscr{L}_{1}$ such that for any $\sigma \in \Sigma_{1}$ and $\left(\alpha_{1}, \beta_{1}, \rho_{1}\right)=L_{1}(\sigma) \in \Sigma_{1}$

$$
h_{\sigma}(z)=\frac{\alpha_{1}}{\sqrt{2 \pi}} \frac{1}{\beta_{1}(1+z)+1-z+\left(1-z^{2}\right) \int_{-1}^{1} \frac{1}{1-t z} \rho_{1}(d t)}\left(z \in U_{1}(0)\right) .
$$

Proof. By Theorem 4. 1, we have an injective mapping $L_{1}$ from $\Sigma_{1}$ into $\mathscr{L}_{1}$ satisfying relation (5.4) and so we have only to show that $L_{1}$ is surjective. Let $\left(\alpha_{1}, \beta_{1}, \rho_{1}\right)$ be any element of $\mathscr{L}_{1}$. We define a new triple $\left(\alpha_{c}\right.$, $\left.\beta_{c}, \rho_{c}\right)$ by

$$
\alpha_{c}=\frac{\alpha_{1}}{\sqrt{2}}, \beta_{c}=\beta_{1} \quad \text { and } \quad \rho_{c}=\varphi\left(\frac{2}{1+\bullet} \rho_{1}\right) .
$$

By noting (4.9), we see from Theorem 3.1 in [9] that there exists a unique bounded Borel measure $\sigma_{c}$ on $[0, \infty)$ such taht 


$$
\begin{aligned}
& \sigma_{c}(\{0\})=0 \text { and } \int_{0}^{\infty}\left(\lambda+\lambda^{-1}\right) \sigma_{c}(d \lambda)<\infty \\
& h_{c}(\xi)=\frac{\alpha_{c}}{\sqrt{2 \pi}} \frac{1}{\beta_{c}-i \xi-i \xi \int_{0}^{\infty} \frac{1}{\lambda-i \xi} \rho_{c}(d \lambda)} \quad\left(\xi \in C^{+}\right),
\end{aligned}
$$

where $h_{c}$ is the outer function of the Hardy weight $\Delta_{c}$ of the form

$$
\Delta_{c}(\xi)=\frac{1}{\pi} \int_{0}^{\infty} \frac{\lambda}{\xi^{2}+\lambda^{2}} \sigma_{c}(d \lambda) \quad(\xi \in \boldsymbol{R}) .
$$

We then define a bounded Borel measure $\sigma$ on $[-1,1]$ by

$$
\sigma=\varphi^{-1}\left(\sigma_{c}\right)
$$

By Lemma 3.2, (5.6) and (5.9), we see that $\sigma \in \Sigma_{1}$. Furthermore, it follows from Lemma 3.1 (i) and (3.12) that

$$
h_{\sigma}(z)=\frac{\sqrt{2}}{1+z} h_{c}\left(i \frac{1-z}{1+z}\right) \quad\left(z \in U_{1}(0)\right) .
$$

By combining this with (5.7) and then noting (5.5), we conclude that $L_{1}(\sigma)=\left(\alpha_{1}, \beta_{1}, \rho_{1}\right)$.

(Q.E. D.)

For each bounded Borel measure $\rho$ on $[-1,1]$ with $\rho(\{-1,1\})=0$, we define a function $\gamma$ on $\boldsymbol{Z}$ by

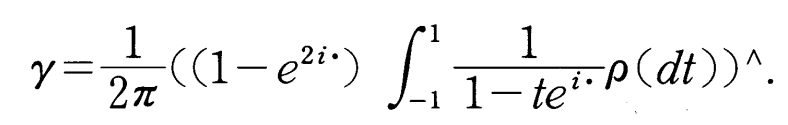

We note that $\gamma$ is well-defined, because

$$
\left|\frac{\left(1+e^{i \theta}\right)\left(1-e^{i \theta}\right)}{1-t e^{i \theta}}\right| \leq 2 \quad \text { for any } t \in(-1,1) \text { and } \theta \in(-\pi, \pi) .
$$

Some properties of $\gamma$ are listed as

Proposition 5.1.

(i ) $\gamma(n)= \begin{cases}0 & \text { for } n \in\{-1,-2, \ldots\} \\ \int_{-1}^{1} t^{n} \rho(d t) & \text { for } n \in\{0,1\} \\ \int_{-1}^{1}\left(t^{n}-t^{n-2}\right) \rho(d t) & \text { for } n \in\{2,3, \ldots\}\end{cases}$

(ii) $\gamma \in l^{1}(\boldsymbol{Z})$

(iii) $\sum_{n=0}^{\infty} \gamma(n)=0$

(iv) $\sum_{n=0}^{\infty}(-1)^{n} \gamma(n)=0$. 
Proof. By the definition of $\gamma$, we have

$$
\gamma(n)=\frac{1}{2 \pi} \int_{-1}^{1}\left(\int_{-\pi}^{\pi} e^{-i n \theta} \frac{1-e^{i 2 \theta}}{1-t e^{i \theta}} d \theta\right) \rho(d t) \quad(n \in \boldsymbol{Z}) .
$$

Hence (i ) follows from the simple fact that for any $t \in(-1,1)$

$$
\int_{-\pi}^{\pi} e^{-i n \theta} \frac{1-e^{i 2 \theta}}{1-t e^{i \theta}} d \theta= \begin{cases}0 & \text { for } n \in\{-1,-2, \ldots\} \\ 2 \pi t^{n} & \text { for } n \in\{0,1\} \\ 2 \pi\left(t^{n}-t^{n-2}\right) & \text { for } n \in\{2,3, \ldots\} .\end{cases}
$$

Since

$$
\sum_{n=2}^{\infty}|\gamma(n)| \leq \int_{-1}^{1}\left(\sum_{n=2}^{\infty}|t|^{n-2}\right)\left(1-t^{2}\right) \rho(d t)=\int_{-1}^{1}(1+|t|) \rho(d t)<\infty,
$$

we have (ii). By (ii), we can take the inverse Fourier transform of (5.10) to get

$$
2 \pi \tilde{\gamma}(\theta)=\left(1-e^{2 i \theta}\right) \int_{-1}^{1} \frac{1}{1-t e^{i \theta}} \rho(d t) \quad \text { for any } \theta \in(-\pi, \pi) .
$$

By taking $\theta=0$ and $\theta=-\pi$ in the above expression, we have (iii) and (iv), respectively.

(Q.E. D.)

Definition 5.2. For each $\sigma \in \Sigma_{1}$, we call a triple $\left(\alpha_{1}, \beta_{1}, \rho_{1}\right)\left(=L_{1}(\sigma)\right)$ or $\left(\alpha_{1}, \beta_{1}, \gamma_{1}\right)$ the first KMO-Langevin data associated with $\sigma$ or $R_{\sigma}$.

We will give a formula concerning the first KMO-Langevin data $\left(\alpha_{1}, \beta_{1}, \gamma_{1}\right)$ associated with a fixed $\sigma\left(\in \Sigma_{1}\right)$.

THEOREM 5.2.

(i ) $\alpha_{1}=2 \sqrt{\int_{-1}^{1} \frac{1-t}{1+t} \sigma(d t)}$

(ii) $\beta_{1}=\sqrt{\left(\int_{-1}^{1} \frac{1-t}{1+t} \sigma(d t)\right)\left(\int_{-1}^{1} \frac{1+t}{1-t} \sigma(d t)\right)^{-1}}$

(iii) $\gamma_{1}(n)=\frac{\alpha_{1}}{(2 \pi)^{3 / 2}}\left(\frac{1}{h_{\sigma}\left(e^{i \cdot}\right)}\right)^{\wedge}(n)-\left(\beta_{1}+1\right) \delta_{n, 0}-\left(\beta_{1}-1\right) \delta_{n, 1} \quad(n \in \boldsymbol{Z})$.

Proof. By noting (4.10), we see from (2.6), (2.7) and (3.5) that

$$
\begin{aligned}
\alpha_{1}^{2} & =(2 \sqrt{2 \pi})^{2} \lim _{r \uparrow 1}\left|h_{\sigma}(r)\right|^{2} \\
& =(2 \sqrt{2 \pi})^{2} \Delta(-\pi) \\
& =4 \int_{-1}^{1} \frac{1-t}{1+t} \sigma(d t),
\end{aligned}
$$


which implies (i ). Similarly, by noting (4.11), we have

$$
\begin{aligned}
\beta_{1}^{2} & =\Delta(-\pi)(\Delta(0))^{-1} \\
& =\int_{-1}^{1} \frac{1-t}{1+t} \sigma(d t)\left(\int_{-1}^{1} \frac{1+t}{1-t} \sigma(d t)\right)^{-1},
\end{aligned}
$$

which implies (ii). By Corollary 4.1 (i ), for a.e. $\theta \in(-\pi, \pi)$,

$$
\begin{aligned}
& \left(1-e^{2 i \theta}\right) \int_{-1}^{1} \frac{1}{1-t e^{i \theta}} \rho_{1}(d t) \\
& =\frac{\alpha_{1}}{\sqrt{2 \pi}} \frac{1}{h_{\sigma}\left(e^{i \theta}\right)}-\beta_{1}\left(1+e^{i \theta}\right)-\left(1-e^{i \theta}\right) .
\end{aligned}
$$

By taking Fourier transform of both hand sides, we have (iii). (Q. E. D.)

REMARK 5.1. We see from (2.4), (3.5) and (4.2) that

(i) $\frac{1}{h_{\sigma}(z)}=\exp \left\{\frac{1}{4 \pi} \int_{-\pi}^{\pi} \frac{e^{i \theta}+z}{e^{i \theta}-z} \log \left(\Delta_{\sigma}(\theta)^{-1}\right) d \theta\right\} \quad\left(z \in U_{1}(0)\right)$

(ii) there exist positive constants $c_{1}$ and $c_{2}$ such that

$$
c_{1} \leq \Delta_{\sigma}(\theta) \leq c_{2} \quad \text { for any } \theta \in(-\pi, \pi)
$$

(iii) $\frac{1}{h_{\sigma}}$ is the outer function of the Hardy density $\Delta_{\sigma}^{-1}$.

\section{$\S 6$. The first KMO-Langevin equation}

Returning to $\S 4$, we will derive a stochastic difference equation describing the time evolution of a real stationary Gaussian process $X=(X(n) ; n \in$ $\boldsymbol{Z})$ on a probability space $(\Omega, \mathscr{F}, P$,$) ; the covariance function R$ takes the form (3.1) with some bounded Borel measure $\sigma \in \Sigma_{1}$.

Let the triple $\left(\alpha_{1}, \beta_{1}, \gamma_{1}\right)$ be the first KMO-Langevin data associated with $\sigma$. By using the normalized Gaussian white noise $\boldsymbol{\xi}=(\boldsymbol{\xi}(n) ; n \in \boldsymbol{Z})$ in (2.14), we will show

\section{THEOREM 6. 1 .}

$$
\begin{array}{r}
X(n)-X(n-1)=-\beta_{1}(X(n)+X(n-1))-\left(\gamma_{1} * X\right)(n)+\alpha_{1} \xi(n) \\
\text { a.s. }(n \in Z) .
\end{array}
$$

Proof By Theorems 4.2 (ii) and 4.3, we have (6.2) $E \in l^{1}(\boldsymbol{Z})$.

Furthermore, by noting Proposition 5.1 (ii), we see from (2.14) that the following two random series are absolutely convergent (a.s.) : for any $n \in \boldsymbol{Z}$

$$
X(n)-X(n-1)+\beta_{1}(X(n)+X(n-1))
$$




$$
\begin{gathered}
=\frac{1}{\sqrt{2 \pi}} \sum_{m=-\infty}^{\infty}\left\{E(n-m)-E(n-1-m)+\beta_{1}(E(n-m)\right. \\
\quad+E(n-m-1))\} \xi(m)
\end{gathered}
$$

On the other hand, it follows from (2.9), (5.10), Proposition 5.1 (ii) and (6.2) that for any $n, m \in \boldsymbol{Z}$

$$
\begin{aligned}
& E(n-m)-E(n-1-m)+\beta_{1}(E(n-m)+E(n-m-1)) \\
& =\int_{-\pi}^{\pi} e^{-i(n-m) \theta}\left\{1-e^{i \theta}+\beta_{1}\left(1+e^{i \theta}\right)\right\} h\left(e^{i \theta}\right) d \theta \\
& \sum_{l=-\infty}^{\infty} \gamma_{1}(n-l) E(l-m) \\
& =\int_{-\pi}^{\pi} e^{-i(n-m) \theta}\left(1-e^{2 i \theta}\right) \int_{-1}^{1} \frac{1}{1-t e^{i \theta}} \rho_{1}(d t) h\left(e^{i \theta}\right) d \theta .
\end{aligned}
$$

Therefore, by substituting (6.5) and (6.6) into (6.3) and (6.4), respectively, we conclude from Corollary 4.1 (i) that for any $n \in \boldsymbol{Z}$

$$
\begin{aligned}
& X(n)-X(n-1)+\beta_{1}(X(n)+X(n-1))+\left(\gamma_{1} * X\right)(n) \\
& =\frac{1}{\sqrt{2 \pi}} \sum_{m=-\infty}^{\infty}\left(\int_{-\pi}^{\pi} e^{-i(n-m) \theta} \frac{\alpha_{1}}{\sqrt{2 \pi}} d \theta\right) \xi(m) \\
& =\alpha_{1} \xi(n) \quad \text { a.s., }
\end{aligned}
$$

which completes the proof of Theorem 6.1.

Definition 6.1. We call the stochastic difference equation (6.1) the first KMO-Langevin equation associated with $\boldsymbol{X}$.

As the converse of Theorem 6.1, we will show

THEOREM 6.2. For each triple $\left(\alpha_{1}, \beta_{1}, \rho_{1}\right) \in \mathscr{L}_{1}$ and each normalized Gaussian white noise $\boldsymbol{\xi}=(\boldsymbol{\xi}(n) ; n \in \boldsymbol{Z})$ on a probability space $(\Omega, \mathscr{F}, P)$, there exists a unique real stationary Gaussian process $\boldsymbol{X}=(X(n) ; n \in \boldsymbol{Z})$ on $(\Omega, \mathscr{F}, P)$ with reflection positivity such that $\boldsymbol{X}$ satisfies the first KMOLangevin equation (6.1); the covariance function of this $\boldsymbol{X}$ coincides with $R_{\sigma}, \sigma=L_{1}^{-1}\left(\left(\alpha_{1}, \beta_{1}, \rho_{1}\right)\right)$.

PROOF By Theorem 5. 1, we get a bounded Borel measure $\sigma=L_{1}^{-1}\left(\left(\alpha_{1}\right.\right.$, $\left.\left.\beta_{1}, \rho_{1}\right)\right)$ in $\Sigma_{1}$ such that the outer function $h_{\sigma}$ associated with $\sigma$ takes the form (5. 4). Choose an $l^{2}(\boldsymbol{Z})$-function $E=\hat{h}_{\sigma}$ as a canonical representation kernel to define a real stationary Gaussian process $\boldsymbol{X}=(X(n) ; n \in \boldsymbol{Z})$ : 


$$
X(n)=(\sqrt{2 \pi})^{-1} \sum_{m=-\infty}^{\infty} E(n-m) \xi(m) .
$$

Then it follows from Theorem 6.1 that this $X$ is our desired process.

To prove the uniqueness of such a process $\boldsymbol{X}$, let $\boldsymbol{Y}=(Y(n) ; n \in \boldsymbol{Z})$ be another real stationary Gaussian process on $(\Omega, \mathscr{F}, P)$ satisfying the same equation (6.1). Fix any $m \in \boldsymbol{Z}$. By multiplying both hand sides of equation $(6.1)$ by $\left(h_{\sigma}(\cdot) e^{-i m \cdot}\right) \sim(n)$ and then summing up with respect to $n$, we can observe from (2.10), Corollary 4.1, (5.10), Proposition 5.1 (ii) and (6.2) that

$$
Y(m)=(\sqrt{2 \pi})^{-1} \sum_{n=-\infty}^{m} E(m-n) \xi(n),
$$

which implies $\boldsymbol{Y}=\boldsymbol{X}$.

Example 6.1. Let $\boldsymbol{X}_{p}=\left(X_{p}(n) ; n \in \boldsymbol{Z}\right)$ be a real stationary Gaussian process with the covariance function $R_{p}$ given in (3.2) $(p \in(-1,1))$. We note that each $\boldsymbol{X}_{p}$ has the simple Markov property, and in particular $\boldsymbol{X}_{0}$ represents a normalized Gaussian white noise $\boldsymbol{\xi}$. The first KMO-Langevin equation of $\boldsymbol{X}_{p}$ takes the simplest from

$$
X_{p}(n)-X_{p}(n-1)=-\beta_{p}^{(1)}\left(X_{p}(n)+X_{p}(n-1)\right)+\alpha_{p}^{(1)} \xi(n)
$$

a.s. $(n \in \boldsymbol{Z})$,

where the pair $\left(\boldsymbol{\alpha}_{p}^{(1)}, \boldsymbol{\beta}_{p}^{(1)}\right)$ was given by (4.21). In case $p=0$, the above form (6.13) for the white noise $\boldsymbol{\xi}$ becomes trivial:

$$
\boldsymbol{\xi}(n)-\boldsymbol{\xi}(n-1)=-(\boldsymbol{\xi}(n)+\boldsymbol{\xi}(n-1))+2 \boldsymbol{\xi}(n)
$$

a.s. $(n \in \boldsymbol{Z})$.

ExAmple 6.2. Let $\boldsymbol{X}=(X(n) ; n \in \boldsymbol{Z})$ be a real stationary Gaussian process with the covariance function $R$ of the form (4.22). It follows from Proposition 5.1 (i) that

$$
\gamma_{1}(n)= \begin{cases}0 & \text { for } n \in\{-1,-2, \ldots\} \\ \rho_{0} q_{1}^{n} & \text { for } n \in\{0,1\} \\ \rho_{0} q_{1}^{n-2}\left(q_{1}^{2}-1\right) & \text { for } n \in\{2,3, \ldots\},\end{cases}
$$

where

$$
\rho_{0}=\frac{2\left(q_{1}-p_{1}\right)\left(p_{2}-q_{1}\right)}{\left(1-q_{1}\right)\left(1+p_{1}\right)\left(1+p_{2}\right)}
$$

We note that (i ) in Example 4.2 is a special case $q_{1}=0$ of (ii) in Example 4.2. Therefore, we see from Theorem 6.1 that $\boldsymbol{X}$ satisfies the following KMO-Langevin equation : 


$$
X(n)-X(n-1)=-\beta_{1}(X(n)+X(n-1))-\left(\gamma_{1} * X\right)(n)+\alpha_{1} \xi(n)
$$

a.s. $(n \in \boldsymbol{Z})$,

where the pair $\left(\alpha_{1}, \beta_{1}\right)$ was given by (4.26) or (4.29). It deserves mention that the second term on the right hand side of equation (6.17) depends upon the whole past of $\boldsymbol{X}$ in case $q_{1} \neq 0$, i.e. $a_{1} \neq 0$.

\section{$\S 7$. Generalized fluctuation-dissipation theorems}

In this final section we will prove a couple of relations between our objects-the first KMO-Langevin data $\left(\alpha_{1}, \beta_{1}, \gamma_{1}\right) \in \mathscr{L}_{1}$, the outer function $h$ of $\boldsymbol{X}$ and other important quantities. The physical meaning of these relations will be explained in detail later (see Remarks 7.2 7.4).

Let $\left(\alpha_{1}, \beta_{1}, \rho_{1}\right)$ be any element of $\mathscr{L}_{1}$ and $\boldsymbol{\xi}=(\boldsymbol{\xi}(n) ; n \in \boldsymbol{Z})$ be a normalized Gaussian white noise. By Theorem 6.2, we obtain a real stationary Gaussian process $\boldsymbol{X}=(X(n) ; n \in \boldsymbol{Z})$ as the unique solution of the first KMO-Langevin equation:

$$
\begin{array}{r}
X(n)-X(n-1)=-\beta_{1}(X(n)+X(n-1))-\left(\gamma_{1} * X\right)(n)+\alpha_{1} \xi(n) \\
\text { a.s. }(n \in Z),
\end{array}
$$

where $\gamma_{1}$ is given by (5.10). This process has the covariance function $R=$ $R_{\sigma} \in l^{1}(\boldsymbol{Z})$, with $\sigma=L_{1}^{-1}\left(\left(\alpha_{1}, \beta_{1}, \rho_{1}\right)\right)$.

We will begin with

LEMma 7.1. The following limit exists :

$$
D \equiv \lim _{N \rightarrow \infty} \frac{1}{2 N} E\left(\left(\sum_{n=0}^{N} X(n)\right)^{2}\right)=\sum_{n=0}^{\infty} R(n)-\frac{R(0)}{2} .
$$

Proof. For any $N \in \boldsymbol{Z}$,

$$
\begin{aligned}
E\left(\left(\sum_{n=0}^{N} X(n)\right)^{2}\right) & =(N+1) R(0)+2 \sum_{n=0}^{N-1}\left(\sum_{m=n+1}^{N} R(n-m)\right) \\
& =(N+1) R(0)+2 \sum_{n=1}^{N}\left(\sum_{l=1}^{n} R(l)\right) .
\end{aligned}
$$

Therefore, we have the assertion, noting that $R \in l^{1}(\boldsymbol{Z})$.

(Q.E. D.)

Definition 7.1. In view of the definition of diffusion constant for the continuous-time case (cf. (2.30) in [10]), the above limit $D$ is called the diffusion constant of the procese $\boldsymbol{X}$.

As a discrete analogue of Theorem 2.1 in [10], we will show

THEOREM 7.1. 
(i) For any $\theta \in(-\pi, \pi)$

$\frac{1}{\beta_{1}\left(1+e^{i \theta}\right)+1-e^{i \theta}+2 \pi \tilde{\gamma}_{1}(\theta)}=\frac{h\left(e^{i \theta}\right)}{2 \lim _{\tau \downarrow \pi} h\left(e^{i \tau}\right)}$.

(ii) $\frac{\alpha_{1}^{2}}{2}=R(0) C_{\beta_{1}, \gamma_{1}}$,

where

$$
C_{\beta_{1}, \gamma_{1}}=\pi\left(\int_{-\pi}^{\pi}\left|\beta_{1}\left(1+e^{i \theta}\right)+1-e^{i \theta}+2 \pi \tilde{\gamma}_{1}(\theta)\right|^{-2} d \theta\right)^{-1} .
$$

(iii) $D=\frac{\alpha_{1}^{2}}{2\left(2 \beta_{1}\right)^{2}}$

(iv) $D=\frac{R(0)}{2 \beta_{1}} \frac{\mathrm{C}_{\beta_{1}, \gamma_{1}}}{2 \beta_{1}}$

(v) $\frac{C_{\beta_{1}, \gamma_{1}}}{2 \beta_{1}}-1=\frac{1}{R(0)} \int_{-1}^{1} \int_{-1}^{1} \frac{1+t}{1-t u} \sigma(d t) \rho_{1}(d u)$.

Proof. By noting (5.11), we see from Corollary 4.1 that

$$
2 \lim _{\theta \downarrow-\pi} h\left(e^{i \theta}\right)=\frac{\alpha_{1}}{\sqrt{2 \pi}} .
$$

Therefore, (i ) follows from Corollary 4.1 (i ), (5.10) and Proposition 5. 1 (ii).

By using Corollary 4.1 ( i ), (5.10) and Proposition 5.1 (ii) again, we see from (2.7) that

$$
\Delta(\theta)=\frac{\alpha_{1}^{2}}{2 \pi}\left|\beta_{1}\left(1+e^{i \theta}\right)+1-e^{i \theta}+2 \tilde{\pi \gamma_{1}}(\theta)\right|^{-2} \quad \text { a.e. } \theta \in(-\pi, \pi) .
$$

By integrating both hand sides with respect to $\theta$, we have (ii).

We now compute the diffusion constant D. By (3.1) and (3.7), we have

$$
\begin{aligned}
& \sum_{n=0}^{\infty} R(n)=\int_{-1}^{1} \frac{1}{1-t} \sigma(d t) \\
& \int_{-1}^{1} \frac{1}{1-t} \sigma(d t)=\frac{1}{2} \int_{0}^{\infty}\left(1+\frac{1}{\lambda}\right) \sigma_{c}(d \lambda) \\
& R(0)=\sigma_{c}([0, \infty)) .
\end{aligned}
$$

Hence, appealing to the result in [10], Theorem 2.1 (iii), we get

$$
\begin{aligned}
D & =\frac{1}{2}\left\{\int_{0}^{\infty}\left(1+\frac{1}{\lambda}\right) \sigma_{c}(d \lambda)-\sigma_{c}([0, \infty])\right\}=\frac{1}{2} \int_{0}^{\infty} \frac{1}{\lambda} \sigma_{c}(d \lambda) \\
& =\frac{\alpha_{c}^{2}}{4 \beta_{c}^{2}}=\frac{\alpha_{1}^{2}}{8 \beta_{1}^{2}}
\end{aligned}
$$


which completes the proof of (iii). (iv) is an immediate consequence of (ii) and (iii).

Now, we proceed to the proof of $(\mathrm{v})$. We first claim

$$
\begin{aligned}
E(0) & =\frac{\sqrt{2 \pi} \alpha_{1}}{1+\beta_{1}+\gamma_{1}(0)} \\
E(n) & =\frac{1-\beta_{1}}{1+\beta_{1}} E(0)-\frac{2 \beta_{1}}{1+\beta_{1}} \sum_{m=1}^{n-1} E(m)-\frac{1}{1+\beta_{1}} \sum_{m=1}^{n} \quad \begin{array}{r}
\left(\gamma_{1} * E\right)(m) \\
\quad(n \geq 1) .
\end{array}
\end{aligned}
$$

By Theorems 4.2 and 4.3 , we have

$$
\frac{1}{2 \pi} \sum_{n=0}^{\infty} E(n) z^{n}=h(z) \quad\left(z \in U_{1}(0)\right) .
$$

In particular,

$$
E(0)=\left.2 \pi h(z)\right|_{z=0} .
$$

Therefore, by taking $z=0$ (resp. $n=0$ ) in Theorem 4.1 (resp. Proposition 5.1 (i )), we get (7.8). Furthermore, by using Theorem 4.1] again, we see from (7.10) that for any $n \in N$

$$
E(n)-E(n-1)=-\beta_{1}(E(n)+E(n-1))-\left(\gamma_{1} * E\right)(n)
$$

and so

$$
\begin{aligned}
E(n)-E(0) & =\sum_{m=1}^{n}(E(m)-E(m-1)) \\
& =-\beta_{1}\left(E(0)+E(n)+2 \sum_{m=1}^{n-1} E(m)\right)-\sum_{m=1}^{n}\left(\gamma_{1} * E\right)(m),
\end{aligned}
$$

which implies (7.9).

Next we prove the key formula

$$
\begin{aligned}
2 \pi R(0) & =\left(1+\frac{\gamma_{1}(0)}{1+\beta_{1}}\right) E(0) \sum_{n=0}^{\infty} E(n)-\frac{4 \pi \beta_{1}}{1+\beta_{1}} \sum_{n=1}^{\infty} R(n) \\
& -\frac{2 \pi}{1+\beta_{1}} \sum_{m=0}^{\infty}\left(\sum_{k=0}^{\infty} R(m+k) \gamma_{1}(k)\right) .
\end{aligned}
$$

By substituting (7.8) and (7.9) into (2.13), we have

$$
\begin{aligned}
2 \pi R(0) & =E(0)^{2}+\frac{1-\beta_{1}}{1+\beta_{1}} E(0) \sum_{n=1}^{\infty} E(n)-\frac{2 \beta_{1}}{1+\beta_{1}} \sum_{n=1}^{\infty}\left(\sum_{l=1}^{n-1} E(l)\right) E(n) \\
& -\frac{1}{1+\beta_{1}} \sum_{n=1}^{\infty}\left(\sum_{l=1}^{n}\left(\gamma_{1} * E\right)(l)\right) E(n) .
\end{aligned}
$$

On the other hand, we see from (2.11), (2.13) and (6.2) that 


$$
\begin{aligned}
\sum_{n=1}^{\infty}\left(\sum_{l=1}^{n-1} E(l)\right) E(n) & =\sum_{l=1}^{\infty}\left(\sum_{m=1}^{\infty} E(l+m)\right) E(l) \\
& =\sum_{m=1}^{\infty}\left(\sum_{l=0}^{\infty} E(m+l) E(l)-E(0) E(m+1)\right) \\
& =2 \pi \sum_{m=1}^{\infty} R(m)-E(0) \sum_{m=1}^{\infty} E(m),
\end{aligned}
$$

and

$$
\begin{aligned}
& \sum_{n=1}^{\infty}\left(\sum_{l=1}^{n}\left(\gamma_{1} * E\right)(l)\right) E(n) \\
= & \sum_{m=0}^{\infty} \sum_{k=0}^{\infty}\left(\sum_{l=1}^{\infty} E(l+m) E(l-k)\right) \gamma_{1}(k) \\
= & \sum_{m=0}^{\infty} \sum_{k=0}^{\infty} \gamma_{1}(k)\left\{\sum_{l=0}^{\infty} E(l+m) E(l-k)-E(m) E(-k)\right\} \\
= & 2 \pi \sum_{m=0}^{\infty}\left(\sum_{k=0}^{\infty} R(m+k) \gamma_{1}(k)\right)-\gamma_{1}(0) E(0) \sum_{m=0}^{\infty} E(m) .
\end{aligned}
$$

And so

$$
\begin{aligned}
2 \pi R(0) & =E(0)^{2}+\frac{1-\beta_{1}}{1+\beta_{1}} E(0) \sum_{n=1}^{\infty} E(n) \\
& -\frac{2 \beta_{1}}{1+\beta_{1}}\left(2 \pi \sum_{n=1}^{\infty} R(n)-E(0) \sum_{m=1}^{\infty} E(m)\right) \\
& -\frac{1}{1+\beta_{1}}\left\{2 \pi \sum_{m=0}^{\infty}\left(\sum_{k=0}^{\infty} R(m+k) \gamma_{1}(k)\right)-\gamma_{1}(0) E(0) \sum_{m=0}^{\infty} E(m)\right\} \\
& =E(0)^{2}+E(0) \sum_{n=1}^{\infty} E(n)+\frac{\gamma_{1}(0)}{1+\beta_{1}} E(0) \sum_{m=0}^{\infty} E(m) \\
& -\frac{4 \pi \beta_{1}}{1+\beta_{1}} \sum_{n=1}^{\infty} R(n)-\frac{2 \pi}{1+\beta_{1}} \sum_{m=0}^{\infty}\left(\sum_{k=0}^{\infty} R(m+k) \gamma_{1}(k)\right)
\end{aligned}
$$

which implies (7.11).

We are now ready to show

$$
\frac{C_{\beta_{1}, \gamma_{1}}}{2 \beta_{1}}-1=\frac{1}{R(0)} \sum_{m=0}^{\infty}\left(\sum_{k=0}^{\infty} R(m+k) \gamma_{1}(k)\right),
$$

which is proved in the following manner.

By (7. 10),

$$
\sum_{n=0}^{\infty} E(n)=2 \pi \lim _{x \uparrow 1} h(x) .
$$

And so by (4.11)

$$
\sum_{n=0}^{\infty} E(n)=\frac{\sqrt{2 \pi} \alpha_{1}}{2 \beta_{1}} .
$$


Combining this with (7.8), we have

$$
\left(1+\frac{\gamma_{1}(0)}{1+\beta_{1}}\right) E(0) \sum_{n=0}^{\infty} E(n)=\frac{\pi \alpha_{1}^{2}}{\beta_{1}\left(1+\beta_{1}\right)} .
$$

On the other hand, by Lemmá 7.1 and Theorem 7.1 (iii),

$$
\sum_{n=1}^{\infty} R(n)=\frac{\alpha_{1}^{2}}{8 \beta_{1}^{2}}-\frac{R(0)}{2} .
$$

Therefore, by combining these with (7.11), we see that

$$
\begin{aligned}
R(0) & =\frac{\alpha_{1}^{2}}{2 \beta_{1}\left(1+\beta_{1}\right)}-\frac{2 \beta_{1}}{1+\beta_{1}}\left(\frac{\alpha_{1}^{2}}{8 \beta_{1}^{2}}-\frac{R(0)}{2}\right) \\
& -\frac{1}{1+\beta_{1}} \sum_{m=0}^{\infty}\left(\sum_{k=0}^{\infty} R(m+k) \gamma_{1}(k)\right)
\end{aligned}
$$

and so

$$
R(0)=\frac{\alpha_{1}^{2}}{4 \beta_{1}}-\sum_{m=0}^{\infty}\left(\sum_{k=0}^{\infty} R(m+k) \gamma_{1}(k)\right),
$$

which, together with Theorem 7.1 (ii), implies (7.12).

Next we claim

$$
\sum_{m=0}^{\infty}\left(\sum_{k=0}^{\infty} R(m+k) \gamma_{1}(k)\right)=\int_{-1}^{1} \int_{-1}^{1} \frac{1+t}{1-t u} \sigma(d t) \rho_{1}(d u) .
$$

By (3.1) and Proposition 5.1 (i ),

the left hand side of (7.14) $=\sum_{k=0}^{\infty}\left(\sum_{m=1}^{\infty} R(m+k)\right) \gamma_{1}(k)$

$$
\begin{aligned}
& =\sum_{k=0}^{\infty}\left(\int_{-1}^{1} \frac{t^{k}}{1-t} \sigma(d t)\right) \gamma_{1}(k) \\
& =\int_{-1}^{1}\left\{\sum_{k=0}^{\infty} \gamma_{1}(k) t^{k}\right\} \frac{\sigma(d t)}{1-t}, \\
& =\int_{-1}^{1}\left\{\left(1-t^{2}\right) \int_{-1}^{1} \frac{\rho_{1}(d u)}{1-t u}\right\} \frac{\sigma(d t)}{1-t},
\end{aligned}
$$

which implies (7.14). Thus, we conclude from (7.12) and (7.14) that ( v) holds.

(Q.E. D.)

Before we go into the explanation of the physical meaning of Theorem 7. 1, we will consider the simplest

Example 7.1. Let $\boldsymbol{X}_{p}$ be the same stochastic process as in Example 6 . 1 , and $D_{p}$ be the diffusion constant of $\boldsymbol{X}_{p}$. By (3.2) and Lemma 7.1,
(7.15) $\quad R_{p}(0)=1$
(7.16) $D_{p}=\frac{1+p}{2(1-p)}$. 
By combining these with (4.21), we see that

(7.19) $\quad \frac{\left(\alpha_{p}^{(1)}\right)^{2}}{2}=R_{p}(0)\left(2 \beta_{p}^{(1)}\right)$

(7.20) $\quad D_{p}=\frac{R_{p}^{(0)}}{2 \beta_{p}^{(1)}}$,

which, together with Theorem 7.1 (ii) or Theorem 7.1 (iv), imply

(7.21) $\quad C_{\beta_{p}^{(1)}, \gamma_{p}^{(j)}}=C_{\beta_{p}^{(1)}, 0}=2 \beta_{p}^{(1)}$.

In addition, we see from (3.2), (4.19) and (4.21) that a remarkable relation between $R_{p}$ and $E_{p}$ holds:

$$
R_{p}(n)=\frac{1+\beta_{p}^{(1)}}{\sqrt{2 \pi} \alpha_{p}^{(1)}} E_{p}(n) \quad(n \geq 0) .
$$

We will return to the general case and give some characterization of the simple Markovian property. As a discrete analogue of Theorem 2.2 in [10], we can see from Theorem 7.1 that

THEOREM 7.2.

(i) $\frac{C_{\beta_{1}, \gamma_{1}}}{2 \beta_{1}} \geq 1$

(ii) The following four statements are equivalent;

(a) $\frac{C_{\beta_{1}, \gamma_{1}}}{2 \beta_{1}}=1$

(b) $\quad \gamma_{1}=0$

(c) $\rho_{1}=0$

(d) $\boldsymbol{X}=\boldsymbol{X}_{p}$ with some $p(\in(-1,1))$.

REMARK 7.1. As we have seen in Theorem 2.2 in [10], the relation (7. 22) characterizes the simple Markovian property for the continuous-time processes. However, this is no longer true for the present discrete-time processes. We will give such an example. Let $\boldsymbol{X}$ be a real stationary Gaussian process discussed in the case (i) of Example 6.2 such that

$$
p_{1}=-p_{2} \text { and } \sigma_{1}=\sigma_{2}=\frac{1}{2\left(1-p_{1}^{2}\right)} .
$$

It then follows from Theorem 4.3 and (4.27) the canonical representation kernel $E$ of $\boldsymbol{X}$ becomes

$$
E(n)=\chi_{(0, \infty)}(n) \sqrt{2 \pi\left(1+p_{1}^{2}\right)}\left(p_{1}^{n}+\left(-p_{1}\right)^{n}\right),
$$

which implies the desired relation 


$$
R(n)=\frac{1}{2\left(1-p_{1}^{2}\right) \sqrt{2\left(1-p_{1}^{2}\right)}} E(n) \quad(n \geq 0) .
$$

Finally we will give three remarks concerning the physical meaning of Theorem 7.1 (cf. [10] for the continuous-time case).

REMARK 7.2. In relation ( $i$ ) in Theorem 7.1, the left hand side denotes a complex mobility of the system $\boldsymbol{X}$ described by equation (7.1), which represents the response of the system $\boldsymbol{X}$ to the external force $\alpha_{1} \xi$. On the other hand, the right hand side is determined by the outer funciton of $\boldsymbol{X}$, which represents the thermal fluctuation of the system in equilibrium without the external force. The relation ( $i$ ) in Theorem 7.1 might be said to be the generalized first fluctuation-dissipation theorem.

Remark 7.3. We are now concerned with relation (ii) in Theorem 7. 1. The fluctuation power of a randow force $\alpha_{1} \xi$ in equation (7.1) is $\frac{\alpha_{1}^{2}}{2}$. While, the positive constant $C_{\beta_{1}, \gamma_{1}}$ is expressed in terms of the drift coefficient representing the systematic part of equation (7.1). And from the physical point of view we can regard $R(0)$ as the absolute constant $k T$, where $k$ and $T$ denote the Boltzman constant and absolute temperature of the system in equilibrium, respectively. This leads us to think of $C_{\beta_{1}, \gamma_{1}}$ as the generalized friction constant, and the relation (ii) itself might be said to be the generalized second fluctuation-dissipation theorem.

Remark 7.4. For the Markov process $\boldsymbol{X}_{\boldsymbol{p}}$ in Example 7.1, we found that the diffusion constant $D_{p}$ is inversely proportional to the friction constant $\beta_{p}^{(1)}$. This relation (7.20) is analogous to the classical Einstein relation valid for the Ornstein-Uhlenbeck Brownian motion with continuous time (see (2.29) in [10]). For this reason, we call relation (7.20) for $\boldsymbol{X}_{p}$ the Einstein relation. In a general system described by equation (7.1) with $\gamma_{1} \neq 0$, however, we found a significant deviation (iv) in Theorem 7.1 from the Einstein relation (7.20) with $\gamma_{1}=0$, and obtained the formula ( $\mathrm{v}$ ) in Theorem 7.1 expressing the degree of such a deviation. In view of the analogous fact in the continuous-time case (Theorem 2.1 in [10]), we call the relation (iv) in Theorem 7.1 the generalized Einstein relation.

\section{References}

[1] Box, G.E.P. and G. M. Jenkins., Time series analysis, Forecasting and Control, Holden-Day, 1976.

[ 2 ] Hoffman, K., Banach spaces of analytic functions, Prentice Hall, Englewood Cliffs, N. J., 1962.

[3] KARHUNEN, K., Über die struktur stationarer zufälliger funktịionnen, Ark. Mat., 1 
(1950), 141-160.

[ 4 ] KuBo, R., Statistical mechanical theory of irreversible processes I, general theory and simple applications to magnetic and conduction problem, J. Phys. Soc. Japan 12 (1957), 570-586.

[ 5 ] MORI, H., B. C. SO and T. OSE., Time-correlation functions of one-dimensional transformations, Prog. Theor. Phys., 66(1981), 1266-1283.

[6] OKABE, Y., On a stationary Gaussian process with T-positivity and its associated Langevin equation and S-matrix, J. Fac. Sci. Univ. Tokyo, Sect. IA 26(1979), 115 $-165$.

[ 7 ] OKABE, Y., On a stochastic differential equation for a stationary Gaussian process with T-positivity and the fluctuation-dissipation theorem, J. Fac. Sci. Univ. Tokyo, Sect. IA 28(1981), 169-213.

[8] OKABE, Y., A generalized fluctuation-dissipation theorem for the one-dimensional diffusion process, Comm. Math. Phys., 98(1985), 449-468.

[9] OKABE, Y., On KMO-Langevin equations for stationary Gaussian processes with T. positivity, J. Fac. Sci. Univ. Tokyo, Sect. IA 33(1986), 1-56.

[10] OKABE, Y., On the theory of Brownian motion with the Alder-Wainwright effect, J. Stat. Phys., 45(1986), 953-981.

[11] OKABE, Y., On the theory of discrete KMO-Langevin equations with reflection positivity (II), to be submitted to Hokkaido Math. J.

[12] OKABE, Y., On the theory of discrete KMO-Langevin equations with reflection positivity (III), in preparation.

Department of Mathematics

Faculty of Science

Hokkaido University 